AN AHP-MLP SXNTHETICAL MODEL

FOR STUDY OF THE INDUSTRIAL STRUCTURE

\author{
YANG JIANMEI \\ South China University of Technology
}

\begin{abstract}
This paper, using AHP method, makes a decision of the prior trades of industry in a county of GD provice during the programming term. Afterward the upper and lower bounds of their output values are determined in combination with the predicted values as the extendion of current status.
\end{abstract}

The upper and lower bounds are added to the constraints of a Mup model. The solution of the MLP model are evaluated under certain criteria, afterwards the upper and lower bounds may be revised untill the solution is satisfied.

In the process of formulating a middle/long term program for the development of county-class industry in our country, the output values of each industrial trade in the programming term usually serve as decision variables, and a ML model is formed under certain constraint conditions. The structure of industry is studied with this model:

There are two ways of building MLP model. One of them is demandguiding model. In this kind of model, the constraint equations are formulated on bases of the termianl demands and input-output analysis in programming term. In this method, work load of revising the input-output coefficients is enormous. An alternative is supply-guiding model, in which constraint equations are built on basis of supply capability of the resources such as energy, funds, manpower, technical level etc. during the programming term.

In this paper, the second way is taken. It often occurs in this way that the number of constraint equations are less than that of the dicision variables. According to the principle of simplex mehtod, in this case, an unreasonable result that output values (decision variables) of some trades equal zero is to be produced.

In fact, development of industry is conditioned by not only the factors which can be quantified such as energy resource, funds, manpower, ect. but also by many other factors which can't. be properly quantified now such as ecölogical effects, social effects, properly quantifical conditioning effect caused by those not-quantified factiors should be reflected by adding constraints of the upper and lower bounds of output values to the MLP model.

The values of the upper and lower bounds are usually predicted 
through extending the present values. In this way, a new strategic concept can hardly be demostrated. In addition, the normal quantified predictive methods mainly suitable for shortterm.

Therefore the problem we have to face is to choose a good method to determine properly the upper and lower bounds of constructure in the programming term using MLP model.

We choose the AHP method and then build an AHP-MLP synthetical model in studying industrial structure of a county of $G D$ province.

AN AHP-MLP SYNTHETICAL MODEL

1. Flowchart For The Model

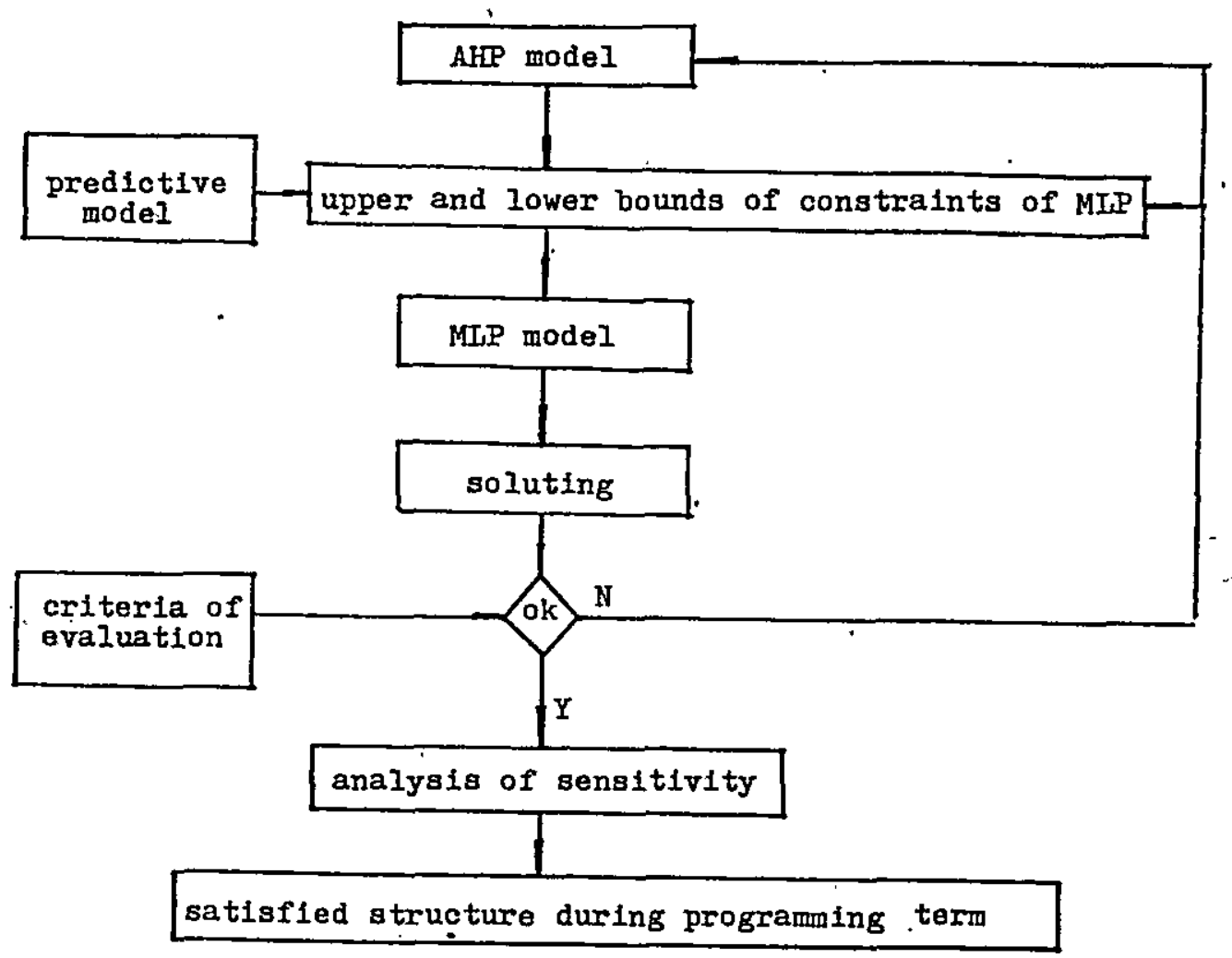

Eigure 1

2. Afr model

Objective of the modelling. 
The purpose of our AHP modelling is ranking the industrial trades of the county according to the degree of importance and determining the initial value of the upper and lower bounds of their output values in combination with the predicted values as the extension of current status.

Hierarchical structure (see Figure 2)

The general rank of the hierarchies

\begin{tabular}{|c|c|c|c|c|c|c|c|c|c|}
\hline I. & $\begin{array}{r}c_{1} \\
0.3032 \\
\end{array}$ & $\begin{array}{c}C_{2} \\
0.1963 \\
\end{array}$ & $\begin{array}{c}c_{3} \\
0.028\end{array}$ & $\begin{array}{c}\mathrm{c}_{4} \\
0.0321 \\
\end{array}$ & $\begin{array}{c}c_{5} \\
0.1862 \\
\end{array}$ & $\begin{array}{c}c_{6} \\
0.1612 \\
\end{array}$ & $\begin{array}{c}c_{7} \\
0.093 \\
\end{array}$ & 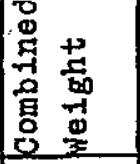 & 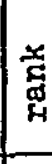 \\
\hline$P_{1}$ & 0.2469 & 0.112 & 0.0161 & 0.0186 & 0.0264 & 0.2034 & 0.563 & 0.188 & 1 \\
\hline$P_{2}$ & 0.1721 & 0.175 & 0.1796 & 0.1666 & 0.0455 & 0.3303 & 0 & 0.1586 & 2 \\
\hline$P_{3}$ & 0.1011 & 0.026 & 0.0344 & 0.2061 & 0.0307 & 0.1231 & 0.145 & 0.0824 & 6 \\
\hline $\mathrm{P}_{4}$ & 0.0424 & 0.057 & 0.016 & 0.0153 & 0.1578 & 0.0664 & 0 & 0.0651 & 8 \\
\hline$P_{5}$ & 0.0172 & 0.015 & 0.06 & 0.0735 & 0.0417 & 0.0431 & 0 & 0.0269 & 9 \\
\hline$P_{6}$ & 0.0605 & 0.207 & 0.2082 & 0.1386 & 0.1293 & 0.0709 & 0.213 & 0.1246 & 5 \\
\hline $\mathrm{P}_{7}$ & 0.1618 & 0.244 & 0.0929 & 0.1183 & 0.0822 & 0.0766 & 0.033 & 0.134 .1 & 4 \\
\hline $\mathrm{P}_{8}$ & 0.0466 & 0.102 & 0.1964 & 0.0519 & 0.1010 & 0.0336 & 0 & 0.0655 & 7 \\
\hline$P_{9}$ & 0.1515 & 0.062 & 0.1964 & 0.2112 & 0.3853 & 0.0524 & 0.045 & 0.1548 & 3 \\
\hline
\end{tabular}

In order to develop the industry of this county rationally, the priority rank of the nine development measures presented in Table 1 is: metallurgical industry (excavation as the main part in it), hydroelectricity generation, industry-foodstuff, industry-forest product, industry-building material, industry-coal mining, industry-medicine, industry-chemical, industry-machine, building industry.

\section{MLP Submodel}

The decision variables

The decision variables are output values of industrial trades of the county in the programing term. They are presented as $X$. $(j=1,2, \ldots, 10)$

objective function 


$$
\begin{aligned}
& \mathrm{P}_{1}=\max \quad \mathrm{z}_{1}=\sum_{j=1}^{10} a_{j} x_{j} \\
& \mathrm{P}_{2}=\min \quad \mathrm{z}_{2}=\sum_{j=1}^{Q} \mathrm{~b}_{j} \mathrm{x}_{j}
\end{aligned}
$$

Where $z_{1}$ is sum of the net output values. of these trades, $z_{2}$ is the transportation cost, a is the rate of net output value of $j$ trade, $b$ is the transportation cost of per 10,000 yuan output value of $\mathbf{j}$ trade.

Constraint conditions

$$
\begin{aligned}
\sum_{j=1}^{10} c_{i j} x_{j} & \leqslant d_{i} \quad(i=1,2 \ldots 6) \\
x_{j} & \leqslant e_{j} \\
x_{j} & \approx f_{j}
\end{aligned}
$$

where $c " c$ are, respectively, the electricity power cost, coal

$$
1 j 6 j
$$

cost, oil cost., demand of fixed assets, demand of manpower, demand of techincians, per 10,000 yuan output value of $j$ trade. $d-d$ are $e$ and $f$ are, respectively, the upper and lower bound $166^{\circ} j \mathrm{j}$

of the output value of $j$ trade.

Method of solution

Turning MLP problem into LP problem, using simlpex method and solve it on computer.

4. Désign' of "the Interface (i'.e." Constraints of upper and Lower Bounds of oütput valúes) between AHP and MLP submodels

It is known from the above-mentioned AHP model that metaliurgical industry (excavation as its chief), hydroelectricity generation industry and foodstuff industry are the prior industrial trades of the county in the programming term. Now we set up the constraint of lower bound to each of them. All the initial values of these lower bounds are decided by experts through discussing the predicted values as the extension current status and the possible speed of development of this trade.

On the other hand, solving the MLP model without any constraints of upper and lower bounds, we can notice in the optimal solution that the forest product industry lincluding making bricks etc.) have very high output values. Hydroelectricity generation industry and coal mining industry have low output values. output values of all other trades are zero.

The about results are caused by the following fact. The forest 
product and building material industries have high rate of net output value and low resources expense. There are not constraints in the aspects such as ecology and market, etc. in our constraints. It demonstrates convincingly that the structure can't be optimized only under now avilable constraints. Therefore we put upper bound constraints upon the forest product and building material industries.

For other trades, the output values in present year are taken as initial values of the lower bound.

We put the above-mentioned initial values of upper and lower bounds into the MLP model and solve it. It appears that the output value of foodstuff industry are too high. Considering the limitation to expanding the market and production capacity, we put an upper bound constraint to the foodstuff industry and solve the model again. When other problems emexge in the new solutions, we revise the upper and lower bounds again, and so on, until a satisfactory structure is obtained under certain criteria.'

\section{Sensitivity Analysis}

In the county's AHP-MLP model of the programming term $a_{j}, b_{j}, c_{j}$ are derived according to the present year's input-output table of the county and $d$ is obtained through the normal quantified i

predictive methods. Generall $Y$ speaking, they are not the exact vaiues of the programing term, therefore we undertake a sensitivity analysis for $a_{j}, b$ and $d_{i} \cdot$

Under the condition that the optimal basis keeps constant, we obtain the variation interval for coefficients of objective function, which are formed through weighting on the net output rate $a$ and the transport cost per 10,000 yuan output value b. and variation interval for the possible supply of resources $d_{j}^{j}$. The shadow prices of them are obtained, too.

The shadow prices of oil in the MLP model is not zero, so"it is effective constraints. The shadow price of oil is 2.09 , i.e. provided oil supply increases for one value unit, the objective function will increase 2.09 value units. 0 il in the county is all bought from beyond the county, so the purchase amount of oil should be increased within the corresponding limits. " This, conclusion turns out quite consistent with the fact that traffic problem is the main difficulty in developing industry of this county.

Having made sensitivity analysis, we work out the proportion of each trade's output value to the total industrial output value so that we gain the optimal structure for the county in the programing term. 
The above presented AHP-MLP model for studing the industrial stucture is only preliminary. The author cansider following points are worth a further study:

1. The synthetical conditioning effect of the factors which now can't be properly quantified should be reflected by adding cönstraints of upper and lower bounds of the decision variables to. the MLI model.

2. Combining AHP method with prediction methods and constructing constraints of upper and lower bounds can not only overcome the difficulties in middle/long-term prediction with the normal quantified predictive methods but also reflect new strategical concepts.

\section{REFERENCE}

Liu Bao, Xu shubo, He Jinsheng (1983), "The AHP-An Implement of Planning Decisłon", Systems Engineering, Vol. 4, No. 6 .

\section{ACKNOWLEDGEMENT}

The author is greatly indebted to Prefessor Li Yijing, Associate professors Fan Zhongzhi and Liao Mingxuan for their warm support. 


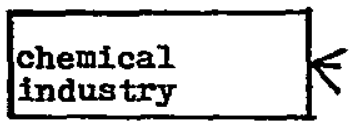

8
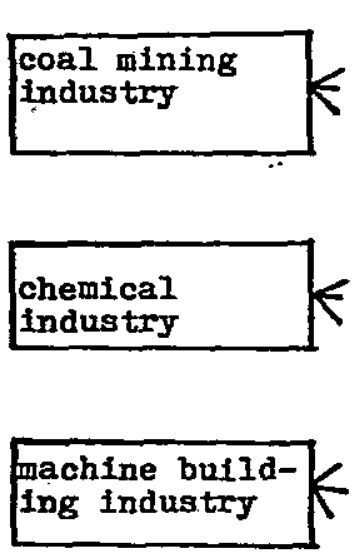

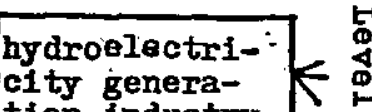
tion industry

\section{chemical}
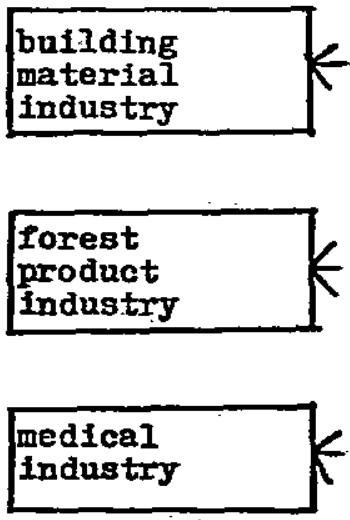

8

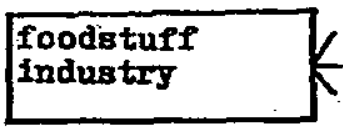

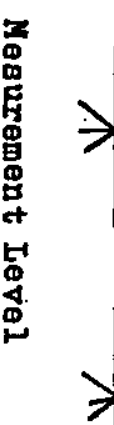

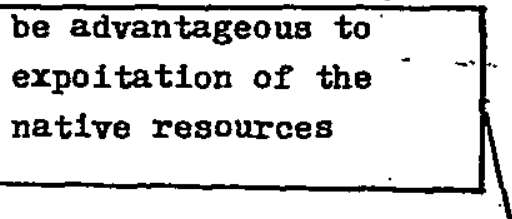

8
0
0
0
4
4
0
0
0
0
0

a high rate of net output value -

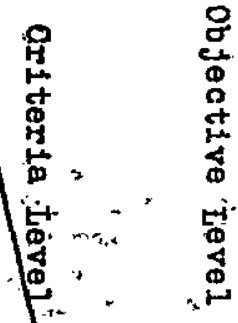

a low transport cost per 10,000 Yuan output value
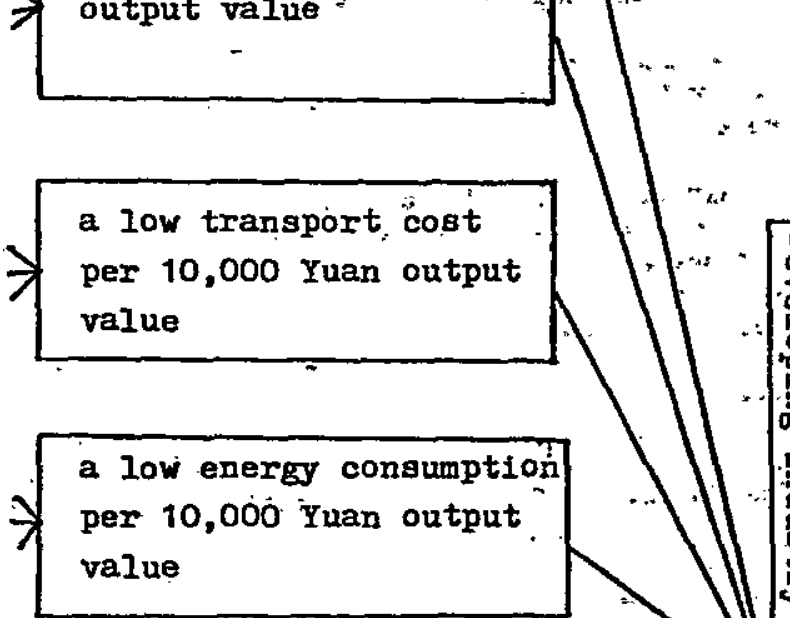

7
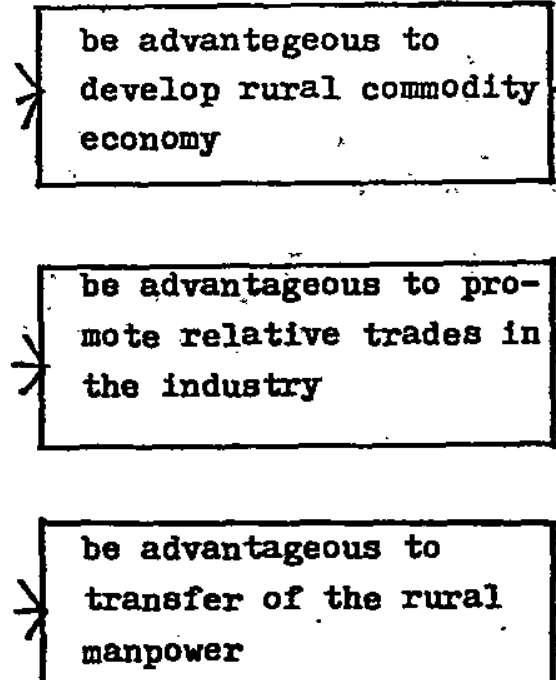

\section{Figure 2}

